\title{
Baryon Spectroscopy Results at the Tevatron
}

\author{
R. Van Kooten \\ for the $C D F$ and $D \emptyset$ Collaborations \\ Department of Physics, Indiana University, Swain West 117, Bloomington, IN 47405
}

\begin{abstract}
.
The Tevatron at Fermilab continues to collect data at high luminosity resulting in datasets in excess of $6 \mathrm{fb}^{-1}$ of integrated luminosity. The high collision energies allow for the observation of new heavy quark baryon states not currently accessible at any other facility. In addition to the ground state $\Lambda_{b}$, the spectroscopy and properties of the new heavy baryon states $\Omega_{b}, \Xi_{b}$, and $\Sigma_{b}^{(*)}$ as measured by the $\mathrm{CDF}$ and $\mathrm{D} \emptyset$ Collaborations will be presented.
\end{abstract}

Keywords: $b$ physics, heavy baryons, spectroscopy

PACS: $14.20 . \mathrm{Mr}, 13.30 . \mathrm{Eg}, 12.39 . \mathrm{Hg}, 12.39 . \mathrm{Jh}, 12.39 . \mathrm{Ki}, 12.40 . \mathrm{Yx}$

\section{INTRODUCTION}

Until within a few days of this conference, the Tevatron has had the highest effective energy collisions in the world, and a great deal of the program focuses on high- $p_{T}$ physics. However, it also provides excellent opportunities to study heavy quark state spectroscopy due to the huge rate and access to large masses. The large rate allows the use of relatively rare decays or rare decays of products to ensure reconstruction of new states with low backgrounds.

Heavy quark hadrons are the hydrogen atom of QCD, and $b$ hadrons offer the heaviest quarks that form bound systems. The study of their spectroscopy provides sensitive tests of potential models, Heavy Quark Effective Theory (HQET [1]), and all regimes of QCD in general, including the non-perturbative regime described by lattice gauge calculations [2]. In addition, decays into heavy quarkonia provides a rich hunting ground for exotics.

There has been somewhat of a "renaissance" of new heavy flavor spectroscopy results these past few years with new data coming from the $B$ factories and the Tevatron. This contribution focuses on new experimental results on heavy $b$ baryons from the CDF and DØ Collaborations running on the Tevatron at Fermi National Laboratory. Results on the spectroscopy of heavy quark mesons at the Tevatron are presented elsewhere in these proceedings [3].

The Tevatron has the capability of producing heavier states not accessible at the $B$ factories running at the $\Upsilon(4 S)$, i.e., the heavy $B$ mesons: $B_{s}^{0}$ ( $\bar{b} s$, the ground state with the spins of the quarks anti-aligned), $B_{s}^{*}(\bar{b} s$, with the spins of the quarks aligned), $B_{c}\left(\bar{b} c\right.$, the ground state), $B^{* *}$ ( $\bar{b} d$, with the quarks having relative orbital angular momentum), and $B_{s}^{* *}\left(\bar{b} s\right.$, with the quarks having relative orbital angular momentum); and the heavy $b$ baryons: $\Lambda_{b}^{0}(b u d), \Sigma_{b}^{(*) \pm}(b u u$ and $b d d)$, and $\Xi_{b}(b s d)$, with many more baryonic states possible from the remaining combinations of quarks.

Charge conjugate modes and reactions are always implied in this contribution.

\section{NEW $b$-FLAVORED BARYONS}

Until recently, the only directly observed $b$ baryon was the $\Lambda_{b}$ [4] (there is indirect evidence for $\Xi_{b}$ through $\Xi$-lepton correlations at LEP [8]). As the Tevatron collects larger and larger data-sets, the prospects improve for observing the rarer $b$ baryons in decay channels providing good triggers. If we consider combinations of only the heavier $b$ quark and the three light quarks $s, d$, and $u$ quarks, multiplets of possible states are shown in Fig. 1, very similar to the multiplets for charm baryons. Double-heavy baryons containing both a charm and $b$ quark, or two $b$ quarks are not shown here.

We can consider a heavy $b$ baryon as a $L=0$ "atomic" system if we take the heavy $b$ quark approximately at rest in the rest frame of the $b$ baryon, and orbited by the diquark of the two light quarks. The two light quarks in the diquark can be anti-aligned, giving diquark spin $s_{q q}=0$ as for the ground state $\Lambda_{b}^{0}(b u d)$. If the spins of the $u$ and $d$ quarks are instead aligned so that $s_{q q}=1$, it results in the more massive $\Sigma_{b}^{0}$ baryon. For the $L=0$ baryons, the total angular momentum is $J=s_{Q}+s_{q q}$, where $s_{Q}$ is the spin of the heavy $b$ quark. The two possibilities are therefore the $J^{P}=\frac{1}{2}^{+}$ 

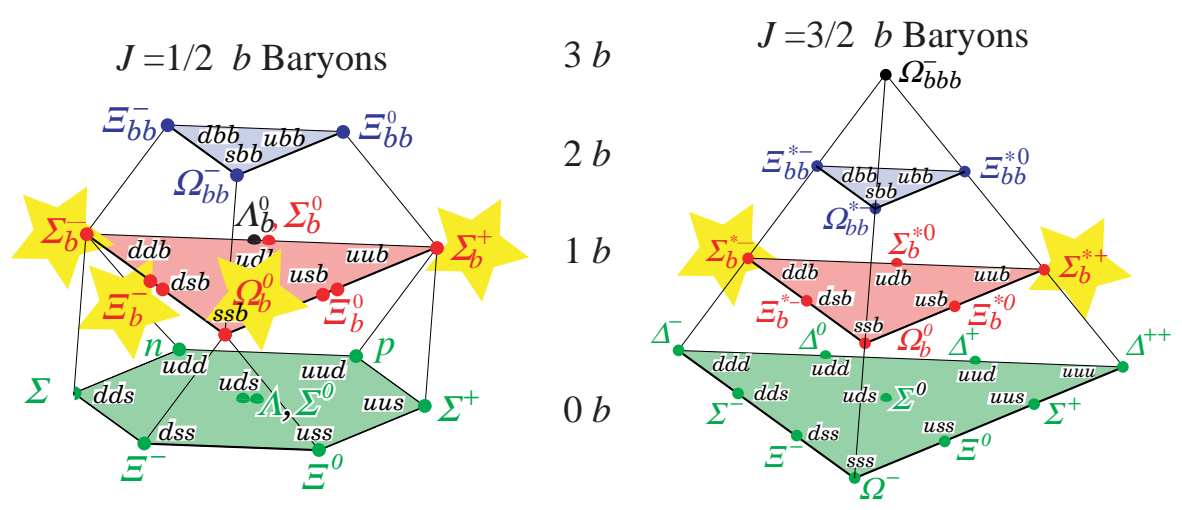

FIGURE 1. Multiplets of possible combinations of the heavy $b$ quark with the $s, d$, and $u$ light quarks with states presented here highlighted (figure adapted from Ref. [4]).

$\Sigma_{b}^{0}$ or the $J^{P}=\frac{3}{2}^{+} \Sigma_{b}^{* 0}$ as shown in Fig. 1 .

Such baryonic systems are valuable for testing predictions from HQET, lattice gauge QCD, potential models, and sum rules. The mass difference $M\left(\Sigma_{b}\right)-M\left(\Lambda_{b}\right)$ probes the energy from the diquark spin alignment, $M\left(\Sigma_{b}^{*}\right)-M\left(\Sigma_{b}\right)$ the equivalent of hyperfine splitting, and $M\left(\Sigma_{b}^{-}\right)[b d d]-M\left(\Sigma_{b}^{+}\right)[b u u]$ the difference due to isospin or effective mass of the $u$ and $d$ quarks. Equivalent measures are also now accessible now that we have evidence for and measurements of the properties of $b$ baryons containing strange quarks such as the $\Xi_{b}(b s d)$ and the $\Omega_{b}(b s s)$.

\section{MASSES OF $\Sigma_{b}^{(*)}$ BARYONS}

In $1.1 \mathrm{fb}^{-1}$ of data, the CDF Collaboration has observed and measured the properties of all four $\Sigma_{b}^{(*) \pm}$ charged baryons [9]. The neutral states $\Sigma_{b}^{(*) 0}$ would decay into $\Lambda_{b}^{0} \pi^{0}$ which is difficult to reconstruct at the Tevatron. Starting with an optimized selection, they form a large, clean sample of $\Lambda_{b}^{0} \rightarrow \Lambda_{c}^{+} \pi^{-}$decays where $\Lambda_{c} \rightarrow p K \pi$. Since the $\Lambda_{b}^{0}$ decays weakly, decay length cuts can be used to further reduce combinatorial background. Since the decay of interest is a strong decay, each of the fitted $N\left(\Lambda_{b}\right)=3180 \pm 60$ candidates is combined with a pion that is constrained to originate from the primary vertex. The largest background is combinatorial with random hadronization tracks being combined with $\Lambda_{b}^{0}$ baryons.

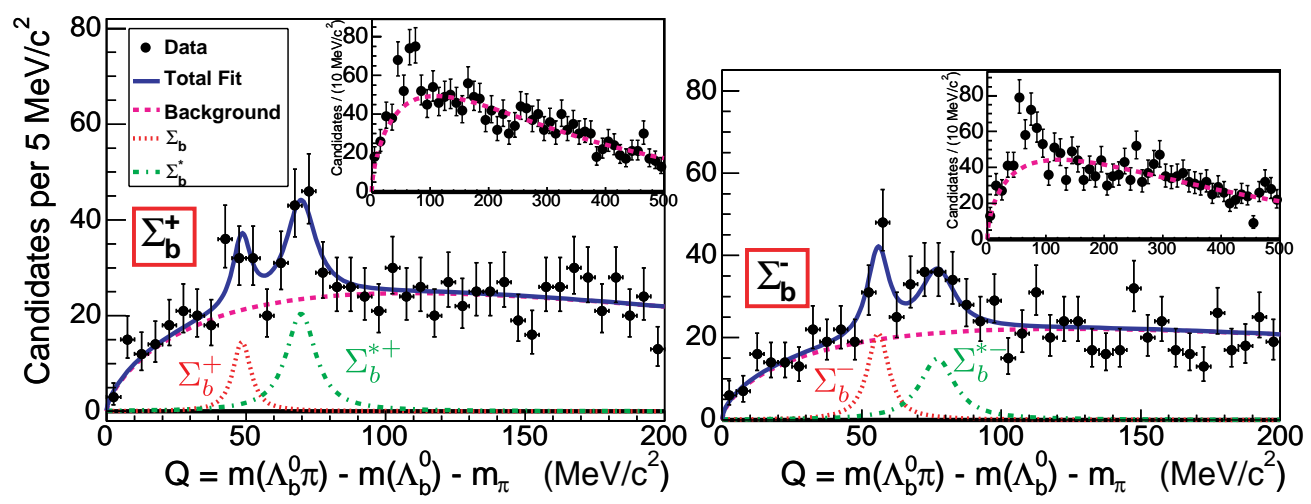

FIGURE 2. $\Sigma_{b}^{(*) \pm}: Q$ value distributions and fits for CDF analysis: top plot for the $\Lambda_{b}^{0} \pi^{+}$subsample that contains $\Sigma_{b}^{+}$and $\Sigma_{b}^{*+}$ and bottom plot for the $\Lambda_{b}^{0} \pi^{-}$subsample that contains $\Sigma_{b}^{-}$and $\Sigma_{b}^{*-}$.

Figure 2 shows the resulting $Q$ value mass difference distributions for the subsequent samples. The different charge sign samples are kept separate and a simultaneous fit performed applying the constraint that the mass splitting between the $J^{P}=\frac{1}{2}^{+}$and $\frac{3}{2}^{+}$states is the same: $M\left(\Sigma^{+}\right)-M\left(\Sigma_{b}^{+}\right)=M\left(\Sigma *^{-}\right)-M\left(\Sigma_{b}^{-}\right)$. Both charge signs $\Sigma_{b}^{-}(b d d)$ and 
$\Sigma_{b}^{+}$(buu) show clear double peak structures, with a 5.2 $\sigma$ significance above background for signal. Using the precision measurement of the $\Lambda_{b}^{0}$ mass [10], the absolute masses are measured to be:

$$
\begin{aligned}
M\left(\Sigma_{b}^{+}\right) & =5807.8_{-2.2}^{+2.0} \pm 1.7 \mathrm{MeV} \\
M\left(\Sigma_{b}^{-}\right) & =5815.2 \pm 1.0 \pm 1.7 \mathrm{MeV} \\
M\left(\Sigma_{b}^{*+}\right) & =5829.0_{-1.8-1.8}^{+1.6+1.7} \mathrm{MeV} \\
M\left(\Sigma_{b}^{*-}\right) & =5836.4 \pm 2.0_{-1.7}^{+1.8} \mathrm{MeV}
\end{aligned}
$$

These values can the be used to calculate various mass splittings of interest as shown in Table 1 and compared to expectations [9]. Theoretical predictions are discussed thoroughly elsewhere in these proceedings [12] and show good agreement with measurements.

TABLE 1. Comparison of measured CDF mass differences to expected values [9].

\begin{tabular}{c|c|c}
\hline \multirow{2}{*}{ Property } & \multicolumn{2}{|c}{ Values $\left(\mathrm{MeV} / \mathrm{c}^{2}\right)$} \\
\cline { 2 - 3 } & Expected & Measured (CDF) \\
\hline Diquark spin alignment & & \\
$M\left(\Sigma_{b}^{+}\right)-M\left(\Lambda_{b}^{0}\right)$ & $180-210$ & $188.1_{-2.2-0.3}^{+2.0+0.2^{\dagger}}$ \\
$M\left(\Sigma_{b}^{-}\right)-M\left(\Lambda_{b}^{0}\right)$ & $180-210$ & $195.5 \pm 1.0 \pm 0.2^{\dagger}$ \\
(isospin averaged) & & \\
$M\left(\Sigma_{b}\right)-M\left(\Lambda_{b}^{0}\right)$ & $194[11]$ & 192 \\
\hline Hyperfine splitting & $10-40$ & $21.1_{-1.9-0.3}^{+2.0+0.4}$ \\
$M\left(\Sigma_{b}^{*}\right)-M\left(\Sigma_{b}\right)$ & $20.0 \pm 0.3[12]$ & \\
\hline Isospin $(u, d)$ diff. & & \\
$M\left(\Sigma_{b}^{-}\right)-M\left(\Sigma_{b}^{+}\right)$ & $5-7$ & $7.4_{-2.4}^{+2.2^{\dagger}}$ \\
\hline Widths & & \\
$\Gamma\left(\Sigma_{b}\right), \Gamma\left(\Sigma_{b}^{*}\right)$ & $\sim 8, \sim 15$ & - \\
\hline
\end{tabular}

$\dagger$ Calculated by author.

\section{WEAKLY DECAYING $b$ BARYONS}

Both CDF and D $\emptyset$ take advantage of the distinctive and easy-to-trigger signature of $J / \psi \rightarrow \mu^{+} \mu^{-}$to generally identify weakly decaying baryons via the quark-flow diagram shown in Fig. 3, and in this case, leading to the observation of new $b$ baryons containing $s$ quarks.

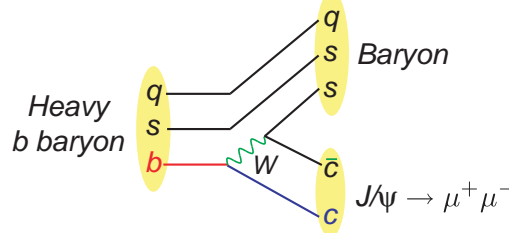

FIGURE 3. Quark flow diagram used in the identification of weakly decaying $b$ baryons at the Tevatron.

\section{Properties of the $\Xi_{b}$ Baryon}

Experiments at the Tevatron has recently discovered the first $b$ baryons containing strange quarks. A possible candidate $\Xi_{b}^{0}(b s u)$ should have dominant decays $\Xi_{b}^{0} \rightarrow \Xi_{c}^{0} \pi^{0}$ and $D^{0} \Lambda$ with neutral states difficult to reconstruct cleanly at the Tevatron. A better candidate is the charged $b$ baryon $\Xi_{b}^{ \pm}$, first observed [13] by the DØ Collaboration in the decay $\Xi_{b}^{ \pm} \rightarrow J / \psi \Xi^{ \pm}$and and also observed [14] CDF Collaboration soon after in the same decay mode plus in the 
additional decay mode $\Xi_{b}^{ \pm} \rightarrow \Xi_{c}^{0} \pi^{ \pm}$. The $\Xi_{b}^{ \pm}$is expected to decay weakly with a lifetime roughly comparable to other $b$ hadrons.

Both collaborations reconstruct the new state in the decay chain $\Xi_{b}^{ \pm} \rightarrow J / \psi \Xi^{ \pm}, J / \psi \rightarrow \mu^{+} \mu^{-}, \Xi \rightarrow \Lambda \pi, \Lambda \rightarrow p \pi$. The reconstruction of the charged $\Xi^{ \pm}$with an average decay length of approximately $5 \mathrm{~cm}$ is challenging in the tracking systems. CDF uses silicon-only tracking, a first for an experiment at a hadron collider, and also modified their vertexing software to include this supplement. $D \emptyset$ reprocesses tracks using special parameter settings to improve the efficiency to reconstruct tracks with high impact parameters.

In $1.1 \mathrm{fb}^{-1}$ of data, $\mathrm{D} \emptyset$ reconstructs $1151 \pm 46 \Xi^{ \pm}$baryons, and in $1.9 \mathrm{fb}^{-1}$ of data, CDF reconstructs $23500 \pm 340$ $\Xi^{ \pm}$baryons. Further selection cuts based on momenta, decay lengths, and vertex quality with the $J / \psi$ are made, with selections optimized on wrong-sign data and signal Monte Carlo samples by DØ, and on a $B^{+} \rightarrow J / \psi K^{+}$control sample by CDF. The resulting invariant mass distributions, including mass constraints to the $J / \psi$ mass, are shown in Fig. 4 with signal yields of $15.2 \pm 4.4$ events (significance of $5.5 \sigma$ above background) and $17.5 \pm 4.3$ events (significance of $7.7 \sigma$ above background) for $\mathrm{D} \emptyset$ and CDF, respectively. D $\varnothing$ also observes a lifetime consistent with expectations for signal candidates. Using this data, CDF measures a mass of $M\left(\Xi_{b}\right)=5792.9 \pm 2.4 \pm 1.7 \mathrm{MeV}$. Using an integrated luminosity of $4.2 \mathrm{fb}^{-1}, \mathrm{CDF}$ has now made a comprehensive reconstruction of $b$ hadrons decaying into $J / \psi$ and have subsequently updated [15] their $\Xi_{b}$ mass measurement (see following subsection for mass distribution), resulting in:

$$
\begin{aligned}
& M\left(\Xi_{b}^{ \pm}\right)=5774 \pm 11 \pm 15 \mathrm{MeV} \mathrm{D} 0, \\
& M\left(\Xi_{b}^{ \pm}\right)=5790.9 \pm 2.6 \pm 0.9 \mathrm{MeV} \text { CDF. }
\end{aligned}
$$

Using this same updated data set, $\mathrm{CDF}$ also measures the relative production rate for $\Xi_{b}$ for $6<p_{T}$ ( $b$ baryon $)<20 \mathrm{GeV}$ as well as the first measurement of the exclusive $\Xi_{b}^{ \pm}$lifetime:

$$
\frac{\sigma\left(\Xi_{b}^{-}\right) \mathscr{B}\left(\Xi_{b}^{-} \rightarrow J / \psi \Xi^{-}\right)}{\sigma\left(\Lambda_{b}^{0}\right) \mathscr{B}\left(\Lambda_{b}^{0} \rightarrow J / \psi \Lambda\right)}=0.167_{-0.025}^{+0.037} \pm 0.012, \quad \tau\left(\Xi^{ \pm}\right)=1.56_{-0.25}^{+0.27} \pm 0.02 \mathrm{ps}
$$
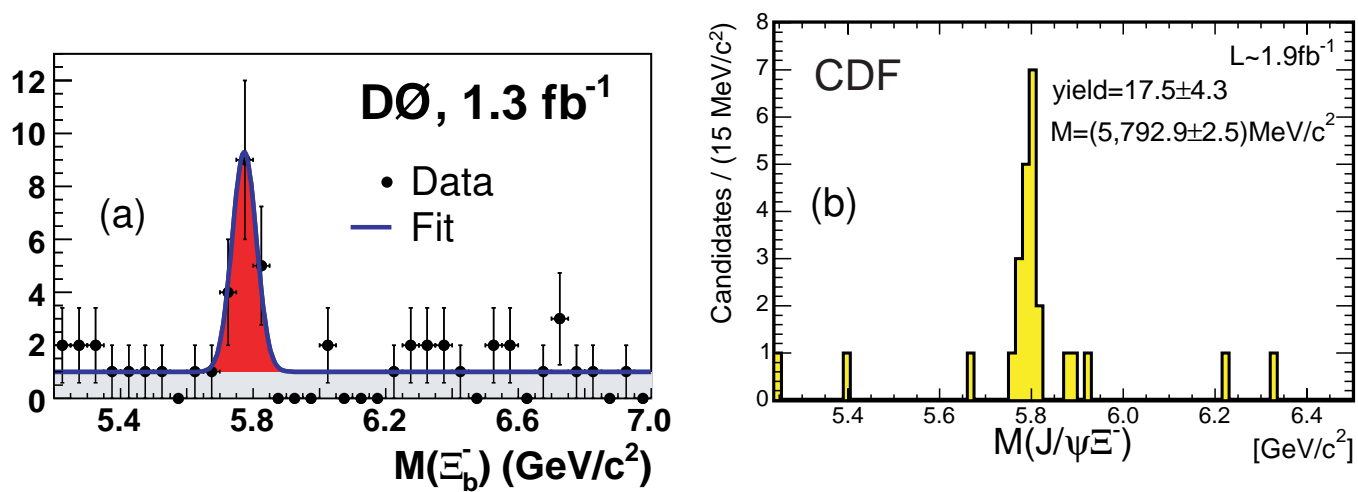

FIGURE 4. $\Xi_{b}^{ \pm}$signal mass peaks in $J / \psi \Xi^{ \pm}$invariant mass distributions following selection cuts for (a) the DØ Collaboration and (b) the CDF Collaboration.

These measurements show good agreement compared to theoretical predictions [16] as shown in Fig. 5.

\section{Properties of the $\Omega_{b}$ Baryon}

Both the D $\emptyset$ and CDF Collaborations have published observations $[17,15]$ of the doubly strange bss baryon $\Omega_{b}$ via the decay chain $\Omega_{b}^{-} \rightarrow J / \psi \Omega^{-}, \Omega^{-} \rightarrow \Lambda K^{-}, \Lambda \rightarrow p \pi^{-}$. DØ reconstructs $\Omega^{-}$baryons as shown in Fig. 6(a) and applies a veto against $\Xi^{-} \rightarrow \Lambda \pi^{-}$decays in this selection. Due to the long decay lengths of the $\Omega^{-}$, special track reprocessing is made to the inclusive $J / \psi$ data set to increase the efficiency for reconstructing tracks with large impact parameters. As shown in Fig. 6(b), in $1.3 \mathrm{fb}^{-1}$ of data, DØ observes $17.8 \pm 4.9 \pm 0.8$ signal candidates after selection cuts, with a statistical significance of $5.4 \sigma$ from the ratio of likelihoods of fits to signal plus background to background alone. When a "trials" factor is included, this significance is $5.05 \sigma$ [18], and remains greater than $5 \sigma$ with systematic checks. 


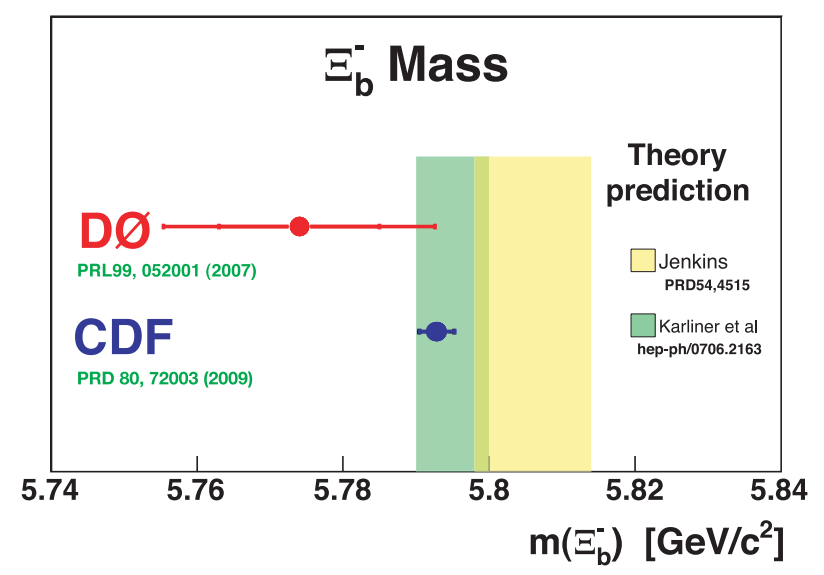

FIGURE 5. Measurements of the $\Xi_{b}^{ \pm}$mass compared to theoretical predictions.

Decay lengths consistent with a weakly decaying $b$ state are observed for the signal candidates, and the production rate with respect to $\Xi_{b}^{-}$is also measured and discussed in the next subsection.
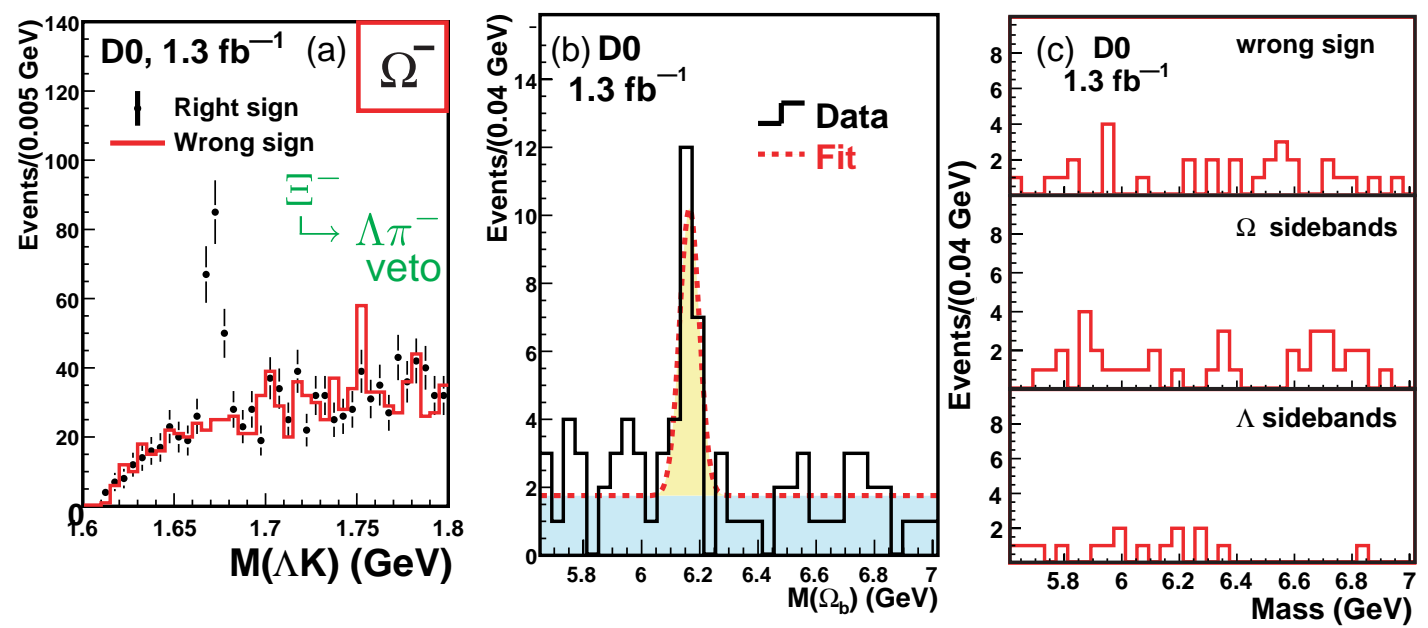

FIGURE 6. (a) Reconstructed $\Omega^{-}$signal before combining with $J / \psi$; (b) $J / \psi \Omega$ invariant mass after selection cuts; and (c) cross checks of backgrounds.

DØ measures a mass of:

$$
M\left(\Omega_{b}^{ \pm}\right)=6165 \pm 10 \pm 13 \mathrm{MeV}
$$

At the time of the publication, the range of expected theoretical values for this state was $5.94-6.12 \mathrm{GeV}$. Since the measured value was greater than expectations, careful checks were made including mass measurements in Monte Carlo samples, generous variation of selection criteria, and a thorough check that the fitted masses of $\Lambda_{b}^{0}$ and $\Xi_{b}^{-}$were consistent with both predictions and prior measurements [4].

As described earlier, CDF uses $4.2 \mathrm{fb}^{-1}$ of data to make a comprehensive reconstruction of $b$ hadrons into $J / \psi$ [15]. As shown in Fig. 7(a), the decay channels $B_{d}^{0} \rightarrow J / \psi K^{* 0}, B_{d}^{0} \rightarrow J / \psi K_{S}^{0}$, and $\Lambda_{b}^{0} \rightarrow J / \psi \Lambda^{0}$ are used as control samples and as sources of cross checks. After selection cuts and demanding decay lengths $c \tau>100 \mu \mathrm{m}$, signals for $\Xi_{b}^{-} \rightarrow J / \psi \Xi^{-}$and $\Omega_{b}^{-} \rightarrow J / \psi \Omega^{-}$are shown in Fig $7(\mathrm{~b})$. A yield of $16_{-4}^{+6}$ events is estimated with a significance of $5.5 \sigma$, determined from invariant mass and lifetime information, likelihood ratios, and toy Monte Carlo tests.

Masses are found by fits to the samples with $c \tau>100 \mu \mathrm{m}$, and lifetimes determined from the signal yield in bins of $c t$ as shown in Fig. 8, resulting in CDF values of:

$$
\begin{aligned}
M\left(\Omega_{b}^{ \pm}\right) & =6054.4 \pm 6.8 \pm 0.9 \mathrm{MeV} \\
\tau\left(\Omega_{b}^{ \pm}\right) & =1.13_{-0.40}^{+0.53} \pm 0.02 \mathrm{ps}
\end{aligned}
$$



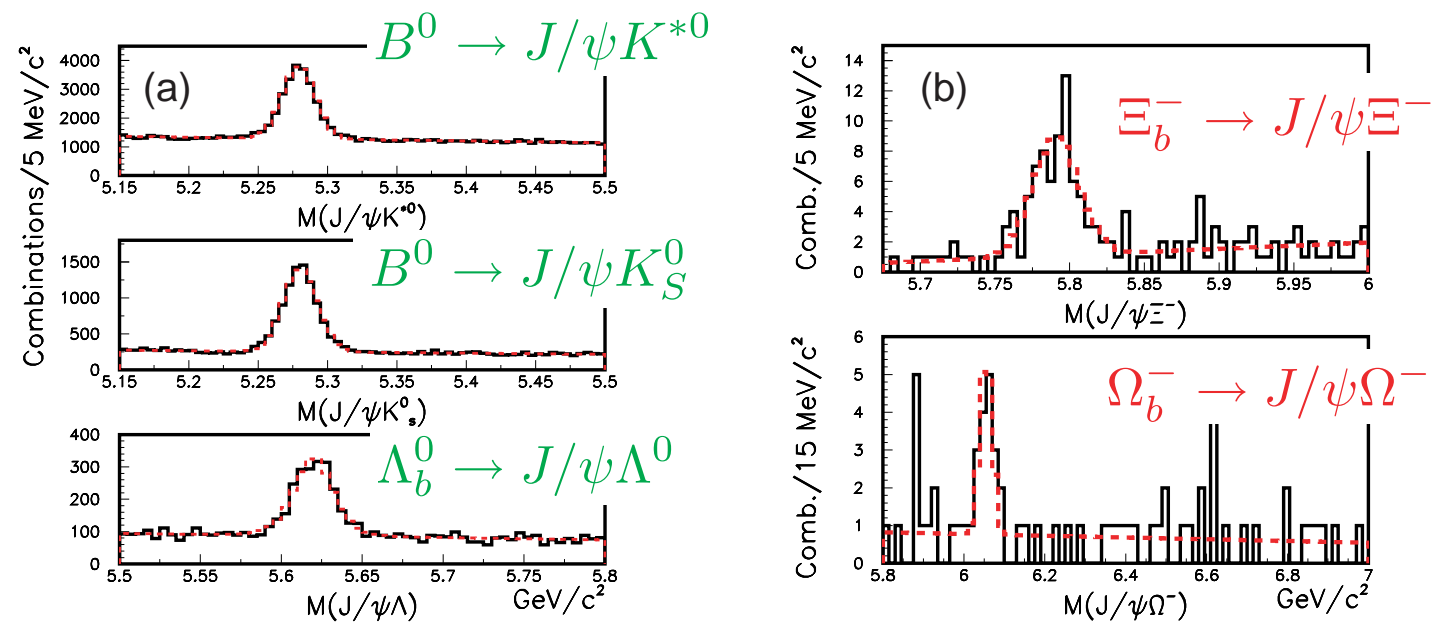

FIGURE 7. For CDF, for $c \tau>100 \mu \mathrm{m}$, (a) Control samples of other $b$ hadrons decaying into $J / \psi$; (b) signal invariant mass peaks for $\Xi_{b}^{0} \rightarrow J / \psi \Xi^{-}$and $\Omega_{b}^{-} \rightarrow J / \psi \Omega^{-}$. The projections of the unbinned mass fit are indicated by the dashed histograms.

where the latter is the first measurement of the $\Omega_{b}$ lifetime. CDF also measures the relative production rate for $6<p_{T}(b$ baryon $)<20 \mathrm{GeV}$ :

$$
\frac{\sigma\left(\Omega_{b}^{-}\right) \mathscr{B}\left(\Omega_{b}^{-} \rightarrow J / \psi \Omega^{-}\right)}{\sigma\left(\Lambda_{b}^{0}\right) \mathscr{B}\left(\Lambda_{b}^{0} \rightarrow J / \psi \Lambda\right)}=0.045_{-0.012}^{+0.017} \pm 0.004
$$
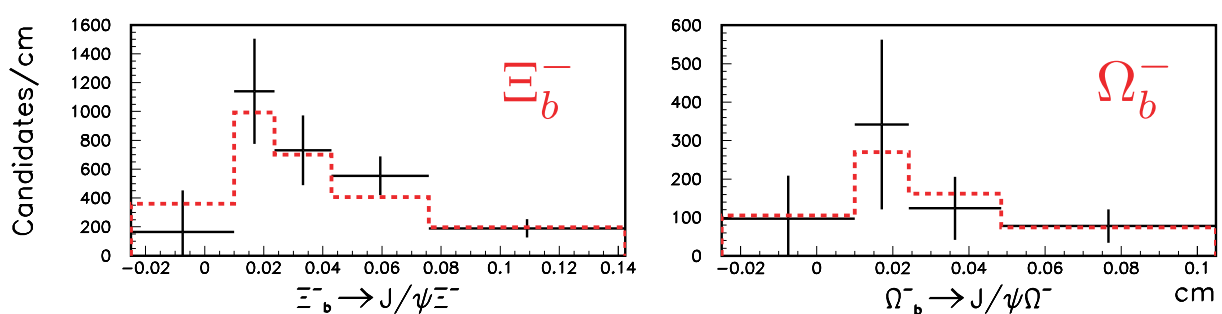

FIGURE 8. For CDF, the solid histograms represent the number of $\Xi_{b}^{-} \rightarrow J / \psi \Xi^{-}$and $\Omega_{b}^{-} \rightarrow J / \psi \Omega^{-}$in each $c t$ bin. The dashed histogram is the fit value in the determination of lifetimes.

\section{Comparison of $\Omega_{b}$ Properties}

Figure 9 compares the mass values measured by CDF and DØ with each other and with a number of more recent model-independent and lattice gauge theoretical predictions for the mass of $\Xi_{b}$ and $\Omega_{b}$ [19]. Comparing the measured mass of the $\Omega_{b}$ between $\mathrm{CDF}$ and $\mathrm{D} \emptyset$ :

$$
M\left(\Omega_{b}^{-}\right)^{\mathrm{DO}}-M\left(\Omega_{b}^{-}\right)^{\mathrm{CDF}}=111 \pm 12 \pm 14 \mathrm{MeV},
$$

which is a significant (approximately $6 \sigma$ ) discrepancy. DØ's largest mass systematic uncertainty is ten times less than this difference. Comparing the signals, there is no significant indication of a mass peak at the CDF mass value in the $\mathrm{D} \emptyset$ mass distribution, and vice versa. The $\mathrm{D} \emptyset$ Collaboration is working on an update of this measurement with an increased data set that may help address this observed difference.

The relative production rates in each case can also be compared. DØ measures [17]:

$$
\frac{f\left(b \rightarrow \Omega_{b}^{-}\right) \mathscr{B}\left(\Omega_{b}^{-} \rightarrow J / \psi \Omega^{-}\right)}{f\left(b \rightarrow \Xi_{b}^{-}\right) \mathscr{B}\left(\Xi_{b}^{-} \rightarrow J / \psi \Xi^{-}\right)}=0.80 \pm 0.32_{-0.22}^{+0.14} .
$$




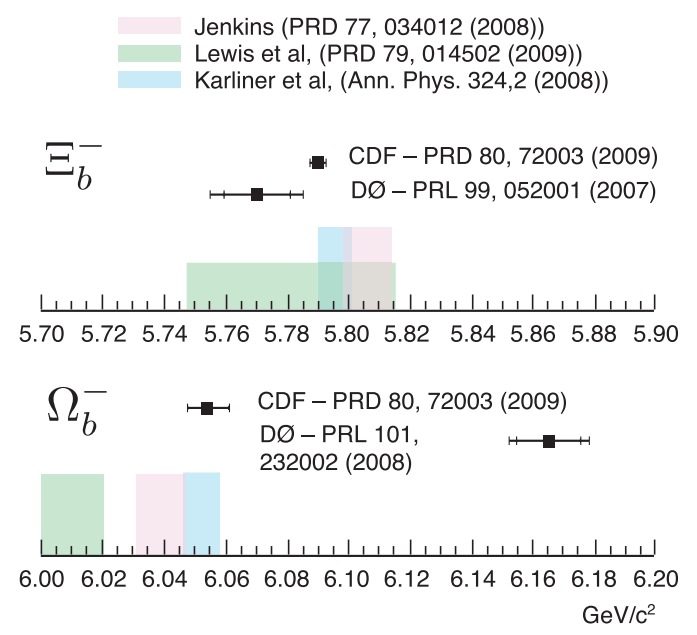

FIGURE 9. Measurement of $\Xi_{b}$ and $\Omega_{b}$ masses compared to each other and to recent theoretical predictions [19].

This can be compared with CDF's relative production rate by taking the measured value of Eq. 6 , the ratio of decay partial widths [20], the CDF lifetime ratio, and an estimate $f\left(b \rightarrow \Omega_{b}^{-}\right) / f\left(b \rightarrow \Xi_{b}^{-}\right) \approx 0.10$ to find:

$$
\frac{\sigma \cdot \mathscr{B}\left(\Omega_{b}^{-} \rightarrow J / \psi \Omega^{-}\right)}{\sigma \cdot \mathscr{B}\left(\Xi_{b}^{-} \rightarrow J / \psi \Xi^{-}\right)}=0.27 \pm 0.12 \pm 0.01
$$

representing a difference of only $1.3 \sigma$ between experiments, assuming Gaussian uncertainties.

\section{CONCLUSIONS AND PROSPECTS}

The resurgence of $b$ hadron spectroscopy (and measurement of properties) continues as new $b$ baryon states are discovered. There is excellent data-theory agreement for most of the new heavy $b$ baryons. However, there exists a greater than $5 \sigma$ discrepancy between $\mathrm{D} \emptyset$ and CDF mass measurements, with the CDF measurement consistent with theoretical predictions. Many of these measurements are providing useful input and comparisons to potential models, HQET, lattice gauge calculations, and other QCD models. It is clear that heavy quark and light quark spectroscopy can benefit each other.

Next good experimental prospects for heavy baryon spectroscopy include the possible observation of double heavy baryons, e.g., $\Xi_{b c} \rightarrow J / \psi \Xi_{c}$ (although heavy quark symmetry would then be lost, see Ref. [21]) and new decay channels such as $\Xi_{b}^{0} \rightarrow \Xi_{c}^{+} \pi^{-}$, among others. The performance of the Tevatron collider has been excellent, with more than $7 \mathrm{fb}^{-1}$ collected to date and expectations to almost double the data-set by the end of running in 2011 . There are very good prospects for increasing the sample sizes of heavier $b$ baryon states and for the discovery of even more states.

\section{ACKNOWLEDGMENTS}

I would like to thank our hosts for their hospitality and an interesting conference. Tallahassee and the Florida State campus are beautiful - I admit a fondness for Spanish moss and was delighted by the surprise of a Ju Ming sculpture on campus.

\section{REFERENCES}

1. N. Isgur and M.B. Wise, Phys. Lett. B 232, 113 (1989); ibid. 237, 527 (1990); Phys. Rev. Lett. 66, 1130 (1991). For some reviews, see: M. Neubert, Phys. Rept. 245, 259 (1994) [arXiv:hep-ph/9306320]; A.V. Manohar and M.B. Wise, Camb. 
Monogr. Part. Phys. Nucl. Phys. Cosmol. 10, 1 (2000).

2. Some example reviews at J.M. Flynn and C.T. Sachrajda, "Heavy quark physics from lattice QCD," Adv. Ser. Direct. High Energy Phys. 15, 402 (1998) [arXiv:hep-lat/9710057]; S.R. Sharpe, "Progress in lattice gauge theory," arXiv:hep-lat/9811006.

3. Kai Yi, "Meson Spectroscopy Results at the Tevatron", these proceedings.

4. C. Amsler et al., Phys. Lett. B667, 1 (2008).

5. T. Matsuki, T. Morii and K. Sudoh, Prog. Theor. Phys. 117, 1077 (2007) [arXiv:hep-ph/0605019].

6. D. Ebert, V. O. Galkin and R. N. Faustov, Phys. Rev. D 57, 5663 (1998) [Erratum-ibid. D 59, 019902 (1999)] [arXiv:hep$\mathrm{ph} / 9712318]$.

7. E. J. Eichten, C. T. Hill and C. Quigg, Phys. Rev. Lett. 71, 4116 (1993) [arXiv:hep-ph/9308337].

8. D. Buskulic et al. [ALEPH Collaboration], Phys. Lett. B 384, 449 (1996);

J. Abdallah et al. [DELPHI Collaboration], Eur. Phys. J. C 44, 299 (2005) [arXiv:hep-ex/0510023].

9. T. Aaltonen et al. [CDF Collaboration], Phys. Rev. Lett. 99, 202001 (2007) [arXiv:0706.3868 [hep-ex]], and references therein for expected values.

10. D. E. Acosta et al. [CDF Collaboration], Phys. Rev. Lett. 96, 202001 (2006) [arXiv:hep-ex/0508022].

11. M. Karliner and H. J. Lipkin, arXiv:hep-ph/0307243; M. Karliner and H. J. Lipkin, Phys. Lett. B 660, 539 (2008) [arXiv:hep-ph/0611306].

12. M. Karliner, B. Keren-Zur, H. J. Lipkin and J. L. Rosner, arXiv:0804.1575 [hep-ph]; M. Karliner, arXiv:0806.4951 [hep-ph], to appear in Proceedings of Flavor Physics and CP Violation (FPCP 2008), Taipei, Taiwan, 5-9 May 2008.

13. V. M. Abazov et al. [DØ Collaboration], Phys. Rev. Lett. 99, 052001 (2007) [arXiv:0706.1690 [hep-ex]].

14. T. Aaltonen et al. [CDF Collaboration], Phys. Rev. Lett. 99, 052002 (2007) [arXiv:0707.0589 [hep-ex]].

15. T. Aaltonen et al. [CDF Collaboration], Phys. Rev. D 80, 072003 (2009) [arXiv:0905.3123 [hep-ex]].

16. E. E. Jenkins, Phys. Rev. D 54, 4515 (1996) [arXiv:hep-ph/9603449]; M. Karliner, B. Keren-Zur, H. J. Lipkin and J. L. Rosner, arXiv:0706.2163 [hep-ph].

17. V. M. Abazov et al. [D0 Collaboration], Phys. Rev. Lett. 101, 232002 (2008) [arXiv:0808.4142 [hep-ex]].

18. “ $\Omega_{b}$ Observation: Frequently Asked Questions", http://www-d0.fnal.gov/Run2Physics/WWW/results/final/B/B08G/faqs.pdf.

19. E. E. Jenkins, Phys. Rev. D 77, 034012 (2008) [arXiv:0712.0406 [hep-ph]];

R. Lewis and R. M. Woloshyn, Phys. Rev. D 79, 014502 (2009) [arXiv:0806.4783 [hep-lat]]; and

M. Karliner, B. Keren-Zur, H. J. Lipkin and J. L. Rosner, Annals Phys. 324, 2 (2009) [arXiv:0804.1575 [hep-ph]].

20. H. Y. Cheng, Phys. Rev. D 56, 2799 (1997) [arXiv:hep-ph/9612223].

21. V. Lyubovitskij, "Decay Properties of double heavy baryons", these proceedings. 